\title{
COMMENTS ON SOLAR CONVECTION
}

\author{
Franz - Ludwig Deubner \\ Institut für Astronomie und Astrophysik \\ der Universität Würzburg \\ Am Hubland, D-8700 Würzburg
}

\begin{abstract}
This contribution discusses observational aspects of the evolution of individual structures of solar convection.

It has been shown, that mesogranulation is a convective phenomenon that fits well into the gap between granulation and supergranulation. Apparently this observation justifies the view that the three members of the granulation family represent sections of a broad continum of convective motions spanning the range of sizes from a yet unknown fraction of $1 \mathrm{Mm}$ to about $50 \mathrm{Mm}$. Nevertheless, power spectra of velocity and brightness fluctuations exhibit three maxima, separated by intervals with significantly less power near $3 \mathrm{Mm}$ and $7.5 \mathrm{Mm}$. Do these gaps give reasons for reconsidering the old idea, that each of the three characteristic scales has its own source layer at a certain depth in the convection zone?

Power spectra of the granular energy distribution near the observational limit of spatial resolution suggest a continuous transfer of kinetic energy to smaller eddies by turbulent decay of the larger scale elements. Morphological studies of granular evolution and a comparison of the observed spectral line bisectors with theoretical predictions seem to disprove this idea. These observations imply either that the turbulent cascade, if it exists, is buried in the spatially unresolved part of the power distribution, or that radiative losses ultimately limit the life time of individual granules on all scales.
\end{abstract}

\section{Introduction}

The condition for convection to occur in a star is well known: it requires that the local adiabatic temperature gradient $|d T / d r|_{\text {ad }}$ be smaller than the temperature gradient found in the ambient medium. In a solar type star with a convection zone in the outer part $(-1 / 3)$ of its envelope, the condition is fulfilled mainly through the decrease of $\gamma$, the ratio of the specific heats $c_{p}$ and $c_{v}$ caused by the ionization of $\mathrm{H}, \mathrm{He}$, and $\mathrm{He}^{+}$in successively deeper layers.

Observations of the sun in white light and in certain spectral lines formed in higher layers of the solar atmosphere have revealed the existence of at least three regimes of convective motions, with different typical sizes, called granulation (1" $-2 ")$, mesogranulation $\left(5^{\prime \prime}-10^{\prime \prime}\right)$, and supergranulation $\left(20^{\prime \prime}-40^{\prime \prime}\right)$. Still larger systems of horizontal surface flows are being searched for, as they are believed to be of fundamental importance in theories of the solar activity cycle (solar dynamo).

3-D numerical simulations of convection based on realistic theoretical models of the solar envelope have made considerable progress in the last years (Nordlund, 1984; Stein et al., 1989; Steffen et al., 1989). It is now possible to compare directly the observed properties of 
convective flows (e.g. spatial scale and life time, r.m.s. velocities and brightness contrast, changes of spectral line profiles) with the corresponding numbers and qualities derived from model simulations (Dravins et al., 1981; Wöhl and Nordlund, 1985; Steffen, 1989). However, due to practical limitations set by the available computing power, typical simulation studies have to contend with 3-D volumes which cover just a few granules in area and a few scale heights in depth.

In this situation, theoreticians presently tend to defend the hypothesis that in the (invisible) interior of the sun we should expect to find at each layer a situation similar to the one studied in the model simulation, which is characterized by extended horizontal flows and narrow downdraft funnels, and which is propagated upward from the bottom layers through essentially all of the convective envelope by selfsimilar expansion and ramification.

In the two chapters of this contribution we want to discuss first, whether the theoretical scenario of a broad continuum of convective scales is indeed supported by observation, with particular attention paid to the newest member of the granulation family (mesogranulation), which was only recently shown to be of genuine convective origin (Deubner, 1989). Later we shall turn to the effects caused by the transition from buoyancy driven structures to inertial eddies, and to the question whether the observations warrant recent speculation about a turbulent decay of convective motions in a Kolmogorovian fashion.

\section{Mesogranulation - the missing link in a uniform spectrum of convective motions?}

Although a mesoscale brightness structure was probably recognized in the photosphere as early as 1932 by Strebel and Thüring, it was only in 1981 through observations of radial velocities near the center of the disc that the existence of a system of vertical motions in the 5 " to $10^{\prime \prime}$ range, presumably of convective origin, was made evident (November et al., 1981). The horizontal counterpart of this flow field became subsequently apparent in studies of proper motions of granules derived from white light high spatial resolution time lapse movies (November et al., 1987; Brandt et al., 1988).

The late discovery of the mesogranulation phenomenon can be understood if the comparatively low contrast of solar structures observed in this range of sizes is taken into consideration: At the photospheric level mesogranular r.m.s. brightness and velocity fluctuations are exceeded by those of granulation, whereas at higher levels (i.e. near the temperature minimum) the 5-min oscillations dominate the velocity signal. Nevertheless, several mesogranulation related effects studied in the past few years testify strongly for the presence of a powerful organized flow pattern.

Two-dimensional measurements of horizontal motions derived with the socalled local correlation technique (November et al., 1987) can be used to estimate the local divergence of the horizontal flow. A comparison of the divergence map with the distribution of magnetic flux reveals that flux concentrations are preferentially located at points or lines of lateral convergence, including alignments which are distinctly smaller than the wellknown supergranular network (Simon et al., 1988). Brandt et al.(1989) have shown, that properties of individual granules like life time, brightness, area, and rate of expansion are statistically correlated with the horizontal divergence in the sense that one would intuitively expect, namely of reinforcement of small scale convection by a similar but larger flow system. At the source points of the horizontal flow, presumably located in local upwellings, an enhanced birth rate of large granules is observed in granule movies.

These kinematic studies have recently been complemented by a comprehensive spatiotemporal analysis of the dynamical behaviour of non-oscillatory flows and brightness 
fluctuations. A range of spatial scales between 1."7 and 200" was investigated spectroscopically at two levels in the lower photosphere simultaneously (Deubner, 1989). Using off center observations on the disc it was confirmed, that the horizontal velocities of approximately 750 $\mathrm{m} / \mathrm{s}$ derived from the changing granular brightness distribution by the local correlation technique do indeed represent a material motion rather than horizontally propagating waves, as was initially suspected. Measurements of the phase lag between the observed brightness and velocity fluctuations, and of their coherence reveal the convective origin of the motions, and the close similarity of mesogranulation to both granulation and supergranulation.

In fact, the phase lag between (upward) velocity and brightness is very close to zero for small spatial scales (granules), and rises only slightly and very smoothly to about $20^{\circ}$ in the range of supergranules. The r.m.s. amplitude of the horizontal velocities decreases almost monotonically from typical granular values (about $2 \mathrm{~km} / \mathrm{s}$ ) to approximately $500 \mathrm{~m} / \mathrm{s}$ for supergranules. It is interesting to note that the ratio of vertical to horizontal r.m.s. velocities decreases nearly linearly with increasing size of the convective elements; the ratio thus seems to obey the scaling law implied by the continuity equation in cylindrical geometry.

The main question to be addressed in this brief discourse on properties of convective motions concerns the existence of several discrete spatial scales of granulation. We do understand, why the different scales are best seen in different layers of the atmosphere, and by using different techniques of observation. Velocity and brightness fluctuations of granulation decay rapidly in the lowest 100 to $200 \mathrm{~km}$ of the photosphere and cannot be seen anymore at higher levels. In contradistinction the Doppler effects due to meso- and supergranular motions are more easily detected in medium strong spectral lines which provide the optimal $S / N$ ratio, i.e. higher in the atmosphere. Finally, the magnetic flux concentrations collected at the cell borders by longer lived large scale motions are most easily seen in the bright cores of chromospheric lines forming the chromospheric network. However, these distinctions would remain even if the spectrum of sizes was perfectly uniform.

On the other hand, the observations of Deubner (1989) indicate gaps in the power and coherence spectra near $4^{\prime \prime}$ and $10^{\prime \prime}$, separating the mesoscale from the larger and smaller size convective elements. A confirmation of these observations by independent spatially twodimensional data is desirable. But the observational task is formidable, because of the wide range (more than two orders of magnitude) of spatial scales and life times of the structures of interest. Also the importance of long lasting high quality seeing to resolve the smallest elements, and of a representative coverage of the solar surface to achieve statistical significance with the larger structures needs to be stressed.

A positive result would necessarily stimulate the question, whether the three different scales can be traced back to certain source regions within the convection zone, as originally suggested by Simon and Leighton (1964), or whether the repeated branching of downdraft funnels, as envisaged by Nordlund (1990), is capable of creating a stable non-uniform spatial power spectrum by some kind of topological effect.

\section{Has turbulent decay of granular convective motions been observed?}

Although life and death of granular convection elements occur right before our eyes, the physical processes which ultimately lead to the conversion of convective energy into heat appear to be even less well determined than the ones discussed in the preceding chapter. The central issue here is, whether convection decays through a cascade of smaller and smaller turbulent elements, and whether the spatial scales involved in this process are already being observed in high resolution photographs and spectrograms. 
This question has recently gained some new momentum through observations obtained by Roudier and Muller (1987) at the Pic du Midi Observatory, which exhibit a break in the areaperimeter relation of granulation in the continuum, and in the histogram of granule areas, at a size of 1."37. A discontinuity at 3" in the one-dimensional(!) power spectrum of granular velocities was presented by Muller (1989). At the short wavelength side of this discontinuity the slope of the observed power spectrum has a value of $-5 / 3$, corresponding closely to the Kolmogorov distribution of energy in a turbulent fluid.

To assess the significance of these observations for our question it is important to realize the preliminary character of the results published so far: The shape of the power spectra we are discussing here is easily altered by a number of effects, all of which need to be carefully considered.

a) Whatever the intrinsic shape of the solar energy distribution may be, the finite resolving power of the instrument and the effects of seeing in the atmosphere and in the spectrograph will inevitably degrade the results, depending on the circumstances. Independent knowledge of the actual point spread function of any given exposure is needed to correct the observed data. Not many observations are available which provide this kind of calibration (cf. Komm and Mattig, 1989; Deubner and Mattig, 1975).

b) Other motions like solar f- and p-modes, and internal gravity waves which are closely connected to the penetration of convective motions into the inertial regime (Deubner and Fleck, 1989), are superposed on, or interfere with the convective velocity and brightness fields. Not all of them can be removed by filtering the data in the wavenumber and frequency domain (Title et al., 1989; Deubner, 1988), since - at least in the most interesting region, the low photosphere - the spectral contribution functions extend across the border line between convectively stable and unstable layers. In this region, the quasistationary primary motions cannot be readily separated from secondary velocity fields, like gravity waves.

c) It is necessary to use two-dimensional spatial information to obtain accurate power spectra. In principle one may convert one-dimensional data under the assumption of azimuthal isotropy to yield two-dimensional spectra. But their statistical stability is often insufficient, and spurious peaks as well as slopes with the wrong gradient are frequently found.

d) But even using a nearly ideal data set which is supposedly free of, or has been corrected for the effects just mentioned, one could hardly expect to retrieve the intrinsic power distribution from the observed spectra, unless the transfer effects of the solar atmosphere are properly taken into account. Since the measured variables (brightness as a proxi of temperature, or velocity) vary not only laterally but also as a function of height in the atmosphere, the inevitable summing of the variables along the line of sight may influence the resulting power law depending on the size and therefore also on the horizontal scale of the structure.

The development of granular structure has first been described in detail by Mehltretter (1978), and more recently by Title et al.(1989), who based their study on a time series of white light pictures obtained from the SOUP data after subsonic filtering. The two studies agree that splitting of granules into two or more fragments is not the only process marking the end of life of selected individual granules. Others disappear by fading, they merge, or they are "squeezed out of existence" by their stronger neighbors. The observed fragmentation does not in general continue on smaller scales; rather, many fragments subsequently become the centers of new full scale granules of the next generation.

The spatial coherence of the brightness and velocity distribution of granules has recently been studied by Deubner (1988) in a high resolution time series of (spatially one-dimensional) 
spectra of the CI 5380 and the FeI 5383 line. He found that velocity and brightness fluctuations (after subsonic filtering!) are well correlated down to element sizes as small as $\sim 0 . " 8$ in the lowest photosphere. At this point the transfer function of the combined atmospheric and spectrographic seeing is expected to decline rapidly due to the comparatively long exposure time of $3 \mathrm{~s}$. But there is no obvious break in the coherence spectra at longer wavelengths, e.g. near the critical value of 3 " reported by Muller (1989), and no substantial change can be detected at 1."37, where Roudier and Muller (1987) have seen a break in the area-perimeter relation.

A very informative pictorial representation of spectral line profiles is the "bisector", i.e. a line which connects the mid-points in between the red and blue wings of the actual profile. This representation contains in a single curve the basic information on the depth dependence of those variables that contribute to an asymmetric shape of the spectral line. It facilitates greatly a direct comparison of observed and theoretical profiles.

Synthetic bisectors have been calculated for this purpose along with the 3-D studies of convection simulation (Dravins et al., 1981; Steffen, 1989) that we have mentioned in the introduction. Of these, the bisectors published by Steffen (1989), which were derived from a stationary model of convection, are the ones that agree best with observed bisectors from high resolution spectra obtained under excellent seeing conditions (Mattig et al., 1989). Synthetic and observed bisectors are displayed in Figures 1 and 2.

The preeminent property of the synthetic profiles is the dependence of the curvature and a monotonic variation of the inclination of the bisectors as a function of continuum brightness. In other words: in Steffen's model, the shape of spatially resolved profiles from the bright center of a granule is systematically and distinctly different from the shape of intergranular profiles. It is not surprising that a stationary model would yield this kind of results; but then, what are the consequences of the impressive similarity of the simulation results with the observed data?

In the range of spatial scales larger than $0 . " 8$ which are well resolved by modem high resolution observations it appears that brightness and velocity are distributed in a well organized fashion, not only with regard to their horizontal distribution but also at any given position as a function of depth in the atmosphere. It is difficult to imagine an atmosphere composed of turbulent eddies with sizes in the vicinity of 1 " or less, which is capable of producing such a smooth and well defined behaviour of the bisectors. It seems therefore unlikely, that turbulent decay of solar convective power occurs within this range of spatial scales.

\section{Conclusions}

The results of extensive theoretical modelling and the data from improved high resolution observations of solar photospheric convection are rapidly converging. There is no doubt that within the limits of present days computer capacity 3-D computer simulations have helped immensely to understand convective processes and to predict their signature on the sun and other stars.

Nevertheless, several questions touching on fundamental subjects like the internal structure of the convection zone, and the detailed energy balance near the border zone to stable stratification, remain to be answered, and further high resolution observations of supreme quality will be necessary along with further refinements of the diagnostic tools to make further progress. It apprears that a sophisticated study of the distribution of convective element sizes may provide answers valuable as a diagnostic of interior structure within the convection zone. Certainly the large body of information available in the form of power-, phase-, and coherence spectra of quasi stationary motions should be utilized to gauge present theories of convection. 

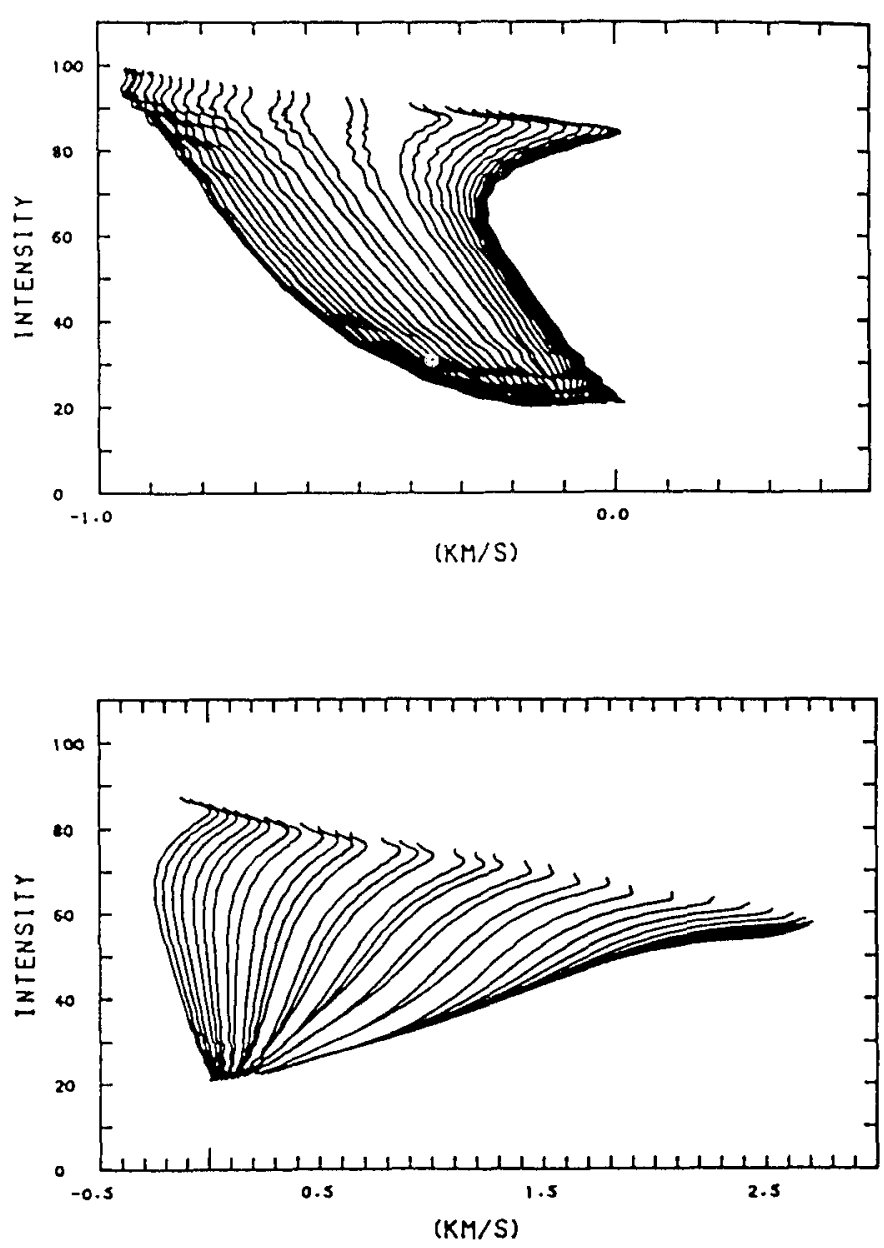

Fig. 1 Top: Series of computed bisectors of a strong FeII line. From left to right, the solar surface area over which the spectrum is integrated is expanded to progressively include contributions of darker granulation elements. Bottom: Similar as in the top figure, but starting with the darkest surface elements (at the extreme right), and advancing by including increasingly brighter elements. Velocities are scaled relative to the laboratory position. Courtesy $M$. Steffen 

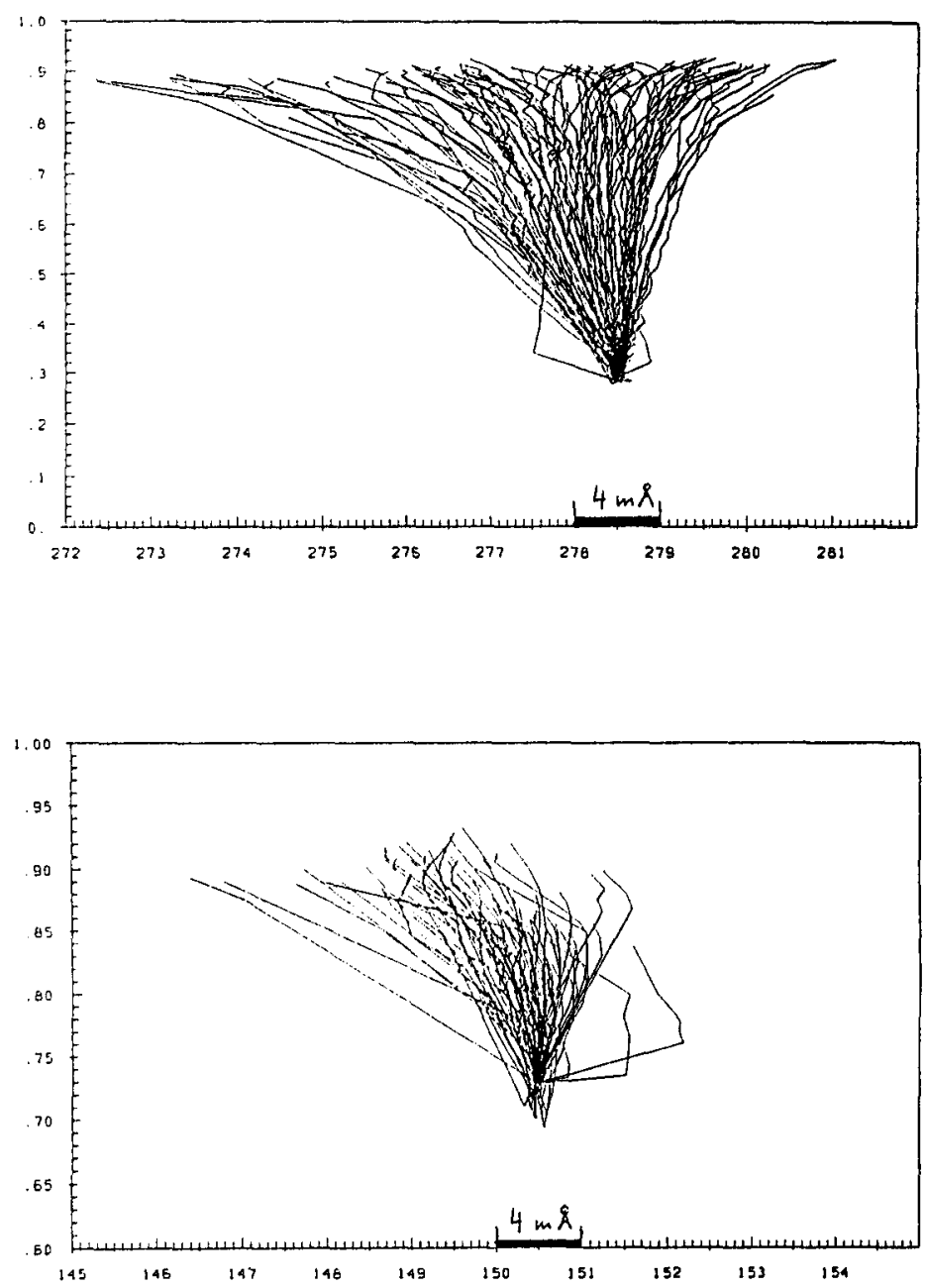

Fig. 2 Top: Series of observed bisectors from profiles of the strong FeI 6494.9 line, covering a range of 16 ". Bottom: Series of observed bisectors from profiles of the weak line FeI 6494.5, as in the top figure. All bisectors have been shifted to the same line core position. Courtesy W. Mattig, A. Hanslmeier, and A. Nesis 


\section{References}

Brandt,P.N., Scharmer,G.B., Ferguson,S.H., Shine,R.A., Tarbell,T.D., Title, A.M.: 1988, Nature 335, 238

Brandt,P.N., Scharmer,G.B., Ferguson,S.H., Shine,R.A., Tarbell,T.D., Title, A.M.: 1989, in High Resolution Solar Observation (O.von der Lühe, ed.) Sacramento Peak,Sunspot N.M. (in print)

Deubner,F.-L.: 1988, Astron.Astrophys. 204, 301

Deubner,F.-L.: 1989, Astron.Astrophys. 216, 259

Deubner,F.-L., Fleck,B.: 1989, Astron.Astrophys. 213, 423

Deubner,F.-L., Mattig,W.: 1975, Astron.Astrophys. 45, 167

Dravins,D., Lindegren,L., Nordlund,A.: 1981, Astron_Astrophys. 96 , 345

Komm,R., Mattig,W.: 1989, in High Resolution Solar Observation (O.von der Lühe, ed.) Sacramento Peak,Sunspot N.M. (in print)

Mattig.W., Hanslmeier,A., Nesis,A.: 1989, in Solar and Stellar Granulation ( R.J.Rutten, G.Severino, eds.) Kluwer Acad. Publ.,Dordrecht. p.187

Mehltretter,J.P.: 1978, Astron.Astrophys. 62, 311

Muller,R.: 1989, in Solar and Stellar Granulation (R.J.Rutten, G.Severino, eds.) Kluwer Acad. Publ., Dordrecht. p.101

Nordlund,A.: 1984, in Small Scale Dynamical Processes (S.Keil, ed.) Sunspot N.M. p.181

Nordlund,A.: 1990, in Solar Photosphere: Structure, Convection, and Magnetic Fields. IAU Symp. 138 (in print)

November,L.J., Toomre,J., Gebbie,K.B., Simon,G.W.: 1981, Astrophys.J. 245, L 123

November,L.J., Simon,G.W., Tarbell,T.D., Title,A.M., Ferguson,S.: 1987, in Theoretical Problems in High Resolution Solar Physics II (G.Athay, D.S.Spicer, eds.) NASA Conf.Publ. 2483 R, p.121

Roudier,Th., Muller,R.: 1987, Solar Phys. 107, 11

Simon,G.W., Leighton,R.B.: 1964, Astrophys.J. 140, 1120

Simon,G.W., Title,A.M., Topka,K.P., Tarbell,T.D., Shine,R.A., Ferguson,S.H., Zirin,H.: 1988, Astrophys.J. 327, 964

Steffen,M.: 1989, in Solar and Stellar Granulation (R.J.Rutten, G.Severino, eds.) Kluwer Acad. Publ., Dordrecht. p. 425

Steffen,M., Ludwig,H.G., Krüß,A.: 1989, Astron.Astrophys. 213, 371

Stein,R.F., Nordlund, $\AA$., Kuhn, J.R.: 1989 in Solar and Stellar Granulation (R.J.Rutten, G.Severino, eds.) Kluwer Acad. Publ., Dordrecht. p.381

Strebel,H., Thüring,B.: 1932, Zs.Ap. 5, 348

Title,A.M., Tarbell,T.D., Topka,K.P., Ferguson,S.H., Shine,R.A.: 1989, Astrophys.J. 336, 475

Wöhl,H., Nordlund,A.: 1985, Solar Phys. 97, 213 